\title{
Cisplatin-Vinblastine Regimen
}

National Cancer Institute

\section{Source}

National Cancer Institute. Cisplatin-Vinblastine Regimen. NCI Thesaurus. Code C63632.

A reg imen consisting of cisplatin and vinblastine used to treat advanced-stage non-small cell lung cancer. 